\title{
Construction of "Sharenting" Reality for Mothers Who Shares Children's Photos and Videos on Instagram
}

\author{
Nur Rafiza Putri ${ }^{1}$ Ali Ar Harkan ${ }^{2}$ Aurora Almarini Khairunnisa ${ }^{3}$, \\ Farisa Nurintan ${ }^{4}$ Moh. Adli Ahdiyat ${ }^{5}$ \\ ${ }^{1}$ Faculty of Political and Social Sciences, University of Indonesia \\ ${ }^{2}$ Faculty of Political and Social Sciences, University of Indonesia \\ ${ }^{3}$ Faculty of Political and Social Sciences, University of Indonesia \\ ${ }^{4}$ Faculty of Political and Social Sciences, University of Indonesia \\ ${ }^{5}$ Faculty of Political and Social Sciences, University of Indonesia
}

\begin{abstract}
The phenomenon of sharing and disclosing intimate information about children in the form of photos, videos, and status by parents through social media is now increasingly widespread. This activity is referred to "sharenting". This activity is commonly done by digital native parents who raise children in digital-first culture. Academically, there are contradictions whether sharenting is part of good parenting or bad parenting. This study aims to understand how is the construction of sharenting reality for mothers who carry out activities to share children's photos and videos on Instagram. This study uses constructivist paradigms, qualitative approaches, constructivism methods, and data collection through interviews with five mothers as informants. The results found that each informant experienced objective reality, symbolic reality, and subjective reality in constructing sharenting reality through three repetitive phases: externalization, objectivation, and internalization. The process of constructing reality that occurs online is explained using the crystallization concept. The three phases of sharenting reality construction are illustrated in the six stages sharenting process: (1) documentation, (2) choosing, (3) editing, (4) giving captions, (5) uploading, and (6) getting responses. In addition to the sharenting process, we also found rules and values of sharenting, family members other than mothers who participated in sharenting, and media other than Instagram that were also used for sharenting. Good or bad parenting depends on the rules made by parents in choosing content that needs to be shared. Good Sharenting is the one that provides benefits by sharing about parenting information with fellow parents to support the child's growth for the better. Meanwhile, sharenting is bad if the parents have violated the privacy of the child so that it can threaten the child's safety.
\end{abstract}

Keywords: Sharenting, Crystallization, Social Construction of Reality, Instagram, Social Media

\section{INTRODUCTION}

\subsection{The Rise of Sharenting Activities Performed by Parents of Digital Natives}

The phenomenon of sharing and disclosing intimate information about children by parents through social media is now increasingly widespread [1]. Information about children shared by parents is in the form of photos, videos and status updates [2]. Mothers are parents who are more willing to upload photos of their children and share information related to childcare topics than fathers [3]. Jomhari et al suggested that mothers prefer to share photos online because communication through photos is easier and faster than through storytelling [1].

Parental activities to share information about children on social networking sites are referred to as sharenting [4]. "Sharenting" is a term that refers to the activities of parents 
sharing information about themselves and their children online, involving both parents and the wider community [4]. The sharenting activity is part of the activity that just emerged when the use of the internet has been rampant and the existence of social media in 00s (Facebook was founded in 2004, Twitter in 2006, YouTube in 2005, and Instagram in 2010). In the analog era (before computers and the internet), the amount of photo production limited by cost and effort. Now, the number of photos taken and distributed increases rapidly. With the good quality of mobile camera, activity takes photos now can always be done. As a consequence, new parents have a tendency to documenting the lives of their babies and share them through various social networking sites [5].

Social media has become a place for parents to share the joy and challenges of being a parent, and become a place to documenting the lives of children in public with increasing frequency, which is almost become a social norm [1]. Instagram nowadays rife with over-sharing information on children, because of the nature of Instagram content in the form of short photos and videos [6].

In addition, the generation is known as digital natives, which is the generation whom was born and grow in the context of developed digital and information technology, most likely already become a parent. In another perspective, they are a generation of millennials who was born in the mid 1980s to the 1990s [7] are now in their late 20 s or early 30 s and might be parents already. They are raising children in digital-first culture, having a tendency to documenting activities digitally. When they were young, before becoming parents, they accessed the online domain based on their own wishes. They can be considered as the last generation of internet users entering the online domain of their own volition, because they are now parents who start sharing information and photos of their babies and young children in the online domain [5].

In the present context, the construction of sharenting is motivated by the presence of digital media. The relation between parenting and digital media that is derived from sharenting contains matters related to mediatization that build new communicative pressure on parents in the present, as they are encouraged to deal with sharenting and specific consequences to follow or reject sharenting [8].

Our pre-research observations found that the choice to carry out this sharenting activity was also related to religious factors. $\mathrm{N}$, a mother with a 1-year-10-month old, revealed that when uploading photos and videos of her child in social media, she concerned about what is referred to as "'ain ailment". According to her statement, 'ain' in the teachings of Islam is the belief that ailment or deprivation can come to someone who exhibits something excessively and creates jealousy to others. According to N, sharenting is an activity to show off the gift of offspring which is actually not owned by everyone, and when uploaded to social media, it can cause jealousy to other parents who do not yet have offspring even though they have sought. If that is the case, other diseases caused by showing off children on social media can turn out to be a disadvantage to the child or family. This is certainly has to be avoided [9].

\subsection{Contradiction on The Meanings of Sharenting: Good or Bad Parenting}

Although sharenting is an activity that is now rife by parents, the view of the activity is contradicted, whether it is part of good parenting or bad parenting. A study that states that sharenting is a good activity explains that sharenting can be a social support mechanism for parents who face caring pressure [10]; become a medium for documenting child growth [11]; help parents break away from feeling isolated [12]; the media shares good ways of nurturing [4]; to fulfill social perceptions as good parents [13]; [10].

On the other hand, studies that assess that sharenting is an activity that is not good to do by parents explains that this can be a violation of children's privacy rights [14]; [4]; [11]; the presence of digital security risks where uploads of children can be used by people who have bad intentions [14]; become a form of child labor because it exploits children to become social media content [2]; and is a form of imaging by parents by utilizing children [2]; [13]; [10]. Based on the findings of several theoretical perspectives that have been presented, we considers that sharenting for parents is an activity that has positive or negative values, so that it can be a good parenting or bad parenting.

Based on the explanation above, we see that there is still a gap that has not been studied, namely in terms of the experience of mothers in carrying out sharenting processes and sharenting phenomena in the Indonesian context. So the purpose of this study is to understand how sharenting reality is constructed for mothers who carry out activities to share children's photos and videos on Instagram. We limited the research problem to how mothers build sharenting reality on the activities of sharing photos and videos he did on Instagram social media. Mothers were chosen as the criteria for the research subjects because they were the parents of those who did sharenting more often than their fathers [3]. The type of upload content shared is limited to photos and videos because the type of content uploaded to the dominant sharenting activity is in the form of photos and videos [2]. Instagram was chosen as the platform used by research subjects (mother) because it is a social media whose native features as a place to upload 
photos and videos are mostly used as sharenting media by parents [6].

\section{LITERATURE REVIEW}

\subsection{Sharenting}

"Sharenting" is a term that refers to the activities of parents sharing information about themselves and their children online, involving both parents and the wider community [4]. Sharenting is part of an activity that only emerged when the use of the internet has become widespread and the existence of social media in the 00s. In the analog era (before computers and the internet), the amount of photo production limited by cost and effort. While now, the number of photos taken and distributed increases rapidly. With the good quality of mobile camera, activity takes photos now can always be done. As a consequence, new parents have a tendency to documenting the lives of their babies and share them through various social networking sites [5]. Social media has become a place for parents to share the joy and challenges of being a parent, and become a place to document the lives of children in public with increasing frequency, which is why these days "sharenting" almost become a social norm [1].

Sharenting is rampant carried out by a generation known as digital natives, which is the generation whom was born and grow in the context of developed digital and information technology, and most likely already become a parent. In another perspective, there are a generation of millennials who was born in the mid 1980s to the 1990s [7] who are now in their late 20s and early 30 s and are becoming young parents. They are raising children in digital-first culture, having a tendency to documenting activities digitally. When they were young, before becoming parents, they accessed the online domain based on their own wishes. They can be considered as the last generation of internet users entering the online domain of their own volition, because now they are parents who start sharing information and photos of their babies and young children in the online domain [5].

\subsection{Crystallization: Social Construction of Reality Facilitated by Social Media}

Crystallization basically refers to a theory initiated by Berger and Luckmann [15], Social Construction of Reality. The theory explains that people and groups interact in a social system which from time to time creates community institutions that are maintained or changed through actions and interactions between humans. Social institutions and communities are objectively visible, but in reality they are built in a subjective definition through an interaction process. The dialectic process is constructed through three moments that occur simultaneously; externalization, objectivation and internalization.

Crystallization is used as a framework for understanding how reality is socially constructed in the era of social media by incorporating network attributes into agenda-setting theory. Crystallization describes the process of information flow and the form of reality in the era of social media. Based on the assumption that individuals psychologically have a basic desire to create a reality together with those who are considered a group, Crystallization positions that individual online social networks will act as neo agenda setters at the first, or second, level. The network will help individuals gather a lot of information produced by various media sources, including old mainstream media and alternative media (social media); and that results from one's personal connection. These individuals will also develop attitudes about cognitive and affective attributes of the information presented to them; which leads to the development of objective and subjective realities [16].

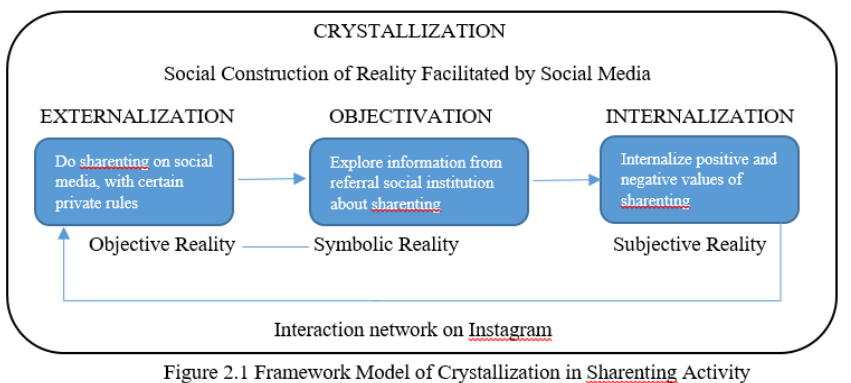

\section{RESEARCH METHODS}

This research uses a constructivist paradigm to understand how mothers build reality from the sharenting activities they do. This study uses a constructivism research strategy because it focuses on the unique experience of each informant, without analyzing in depth the background of the informant [17]. In this study, those who will be the subject of research are mothers who have Instagram social media accounts and share children's photos and videos on Instagram social media. While the object of research in this study is the construction of sharenting reality for mothers who share children's photos and videos on Instagram social media. Data collection in this study uses semi-structured interview techniques. The sampling is purposive sampling - homogeneous sampling that aims to reach a homogeneous sample, that is, samples whose units have the same characteristics (or very similar).

To answer the purpose of the study, we compiled the criteria for the informant. The research conducted is to understand sharenting construction so that the informant must be someone who (1) performs sharenting activities on 
Instagram in the form of photos and videos, and (2) uploads them in feeds and/or Instagram Story. When conducting pre-research observations, we found that sharenting was done with or without showing the child's face, and we wanted to know the views of the two categories, so the next criteria were (3) mothers upload content showed or did not show the child's face. To find out the interactions that occur online on Instagram, we set criteria that informants must (4) get likes and/or comments in their sharenting uploads. We need to understand the thoughts of mothers who are classified as digital natives, therefore the last criteria are (4) mothers aged 19-35 years and have a child with maximum age of 6 years old.

\section{RESULTS}

We conducted interviews with five informants face to face and by phone. As an illustration, the informants have undergraduate and graduate education backgrounds. Four informants are working mothers, while one informant is a housewife. Five informants were chosen for reasons of accessibility, in addition to adjusting to the criteria of the informants. Informant 1 was LS, 23 years old. She is a housewife who has a 1 year old son. Informant 2 is MA, a 27-year-old mother. MA has a daughter aged 20 months, and currently MA works as a private employee. Informant 3 is AS, 34 years old. AS is a civil servant, and currently AS has 6 years old daughter. Informant $4, A B$, is a mother with one baby aged 3 months. $\mathrm{AB}$ is a private employee who is currently 27 years old. Informant 5 is $\mathrm{MC}$ who is a 31 year old mother. MC has a 4 year old boy. Currently MC is a private employee.

We found that each informant construct sharenting reality through three repetitive phases: externalization, objectivation, and internalization, involving three perspectives of reality: objective, symbolic, subjective reality. Informants listened to every word which is told by public figures, professional psychologists, and fellow mothers related to sharenting, in a phase called objectivity. The knowledge gained, coupled with the socio-cultural background, contributes to constructing informants' views about positive and negative values in sharenting activities; in the phase of internalization. The knowledge gained from social institutions in the environment of informants and the values internalized in the informants is then reflected in the activities of sharing photos and videos on social media as a form of informants' adjustment to society. Externalization as a form of adjustment to objectivation and internalization is carried out subjectively by each informant with the background of sharenting goals and rules regarding content that may or may not be shared.

The construction of mothers in sharenting reality involves social media. Mothers interact not only offline, but also online with parties who are considered as agents of socialization. This construction process is explained by using Crystallization which describes the process of information flow and the form of reality in the era of social media. Externalization, objectivation, internalization are depicted in the Sharenting Process which consists of six stages (not all informants go through the six stages). The six stages are (1) documentation, (2) choosing, (3) editing, (4) captioning, (5) uploading, (6) getting responses.

Documenting. First step in the sharing process is documenting. Informants take pictures and or videos, using a cellphone, of the moments or activities of the child. Documenting is not only done by themselves but also by close relatives such as husbands and grandmothers. Documentation is done since pregnancy, birth, and or after birth. For example, AS captures children's activities which she thinks are funny, or in his personal designation "nyampah", such as compiling children imitating beauty vloggers, salon workers, singers, and presenters.

In the documentation process, informants have different motivations. AS documents children in order to record humorous moments. AB who document the intimacy of her son and his father as material to make a positive message about father's parenting role. What was recorded by mother at this stage of documentation reflects a rule about what is permissible or not should be enshrined as documentation. In social construction of reality, the activity of mother documenting children is a form of externalization, in which individuals have certain values which have been internalized regarding sharenting activities. The rules is a manifestation of the view of positive and negative values towards the sharenting that is owned by mother.

Choosing. Not all of the documentation is uploaded to Instagram. Mothers selected the photos and videos to determine one or several that could eventually be uploaded. In this process also applied the sharenting rules. For example, LS does not upload photos showing her child's face to Instagram friends publicly. Photos or videos that show children's faces can only be shared in close friendships by utilizing the close friends feature on Instagram Story. LS also avoids uploading documentation showing the body or when the child is not wearing clothes.

Meanwhile, for photos and videos that were not selected to be uploaded on Instagram social media, the informants applied different treatments. US chooses to backup documentation from the handphone to be stored on private hard drive or on Google Drive. Whereas MA keeps photos and videos on the cellphone. There are also informants who send photos and videos that are not uploaded on Instagram to the family WhatsApp group. 
As the rules at the documentation stage are a reflection of the internalization of the good and bad values of the sharenting that mother has, how the mothers choose which photos and videos to upload or not upload on Instagram is also a manifestation of the construction of sharenting reality to mother. Mothers have a subjective reality of how appropriate photos and videos are to be uploaded and not, so the selection process is done before uploading.

Editing. After taking pictures in the form of photos or videos, there is an editing process that the informants make to the photos or videos chosen to be shared on social media. Editing photos or videos chosen by all informants in different ways and intentions.

LS, MA, and AB add stickers or gifs, with the aim of covering the child's body parts that seems to be too open, or to make the content to look funnier. MA added a photo filter and used a photo grid to put a photo of his child and a celebrity's child in one post that aimed to show that the child was similar to the celebrity's child. AS edits sizes of the photo, such as crop and resize, and MC sometimes uses the Photo Wander editing application.

Captioning. The next step is to prepare the captions. Informants always write captions on photos or videos uploaded to social media. The captions made by informants are quite diverse. LS uses English in writing in each post. $\mathrm{AS}, \mathrm{AB}$ and $\mathrm{MC}$ write a caption that contains a story that matches the image uploaded if it has special meaning, or writes "funny" jokes, or "poor" stories that actually don't match the content. $\mathrm{AB}$ writes a caption with the contents of positive messages regarding the role of father in parenting, whose purpose was to share and hope to inspire others. MA, $\mathrm{AB}$ and $\mathrm{MC}$ said that sometimes they prefer adding hashtags or emoticons. Some other times, they post without any captioning.

Uploading. The fifth step is uploading photos or videos on Instagram. However, there are times when they upload photos or videos of their child spontaneously using the Instastory feature because they just capturing a moment when their child is acting funny like what $\mathrm{MC}$ and $\mathrm{AB}$ do. The activity of uploading children's documentation on social media is based on diverse motivations and goals. MA and AS upload photos of children on Instagram because they think that Instagram is one of the good media for storing photos and videos. However, there are differences between MA and AS in looking at the activity of uploading children's photos or videos on Instagram. MA uploads a photo of her child on Instagram to get a response from her friends.

Unlike MA, AS uploads photos or videos of their children solely for entertainment and does not want to see the responses of others. In addition, uploading activities are also based on positive and negative views of the mother about sharenting activities, so that a mother has certain restrictions or rules regarding the content about the child that will be uploaded on Instagram. The content that is most uploaded by the mothers is the moment when children are acting funny and precious moments when a new child is born or when the child first goes somewhere.

Getting responses. After uploading the sharenting content, the informants got a response from followers on their Instagram. Responses given by followers to the post are in the form of likes, and in the form of praise comments such as "Funny" or "Smart", comments in the form of emoticons, and appreciation of milestones. As revealed in the LS informant's interview, the appreciation of the milestone is a compliment to the growth of the child when the child is able to crawl, walk, talk, and other growth and development processes. The comments given by followers of MA such as when informants upload about children's clothes, followers comment on the clothes worn by children. Comments given by followers of informants are also not always about post, as AS said that the response was just "nyampah", funny, not such a serious response. This response was obtained because the followers of the informants were friends of the informant themselves and were already familiar. There are also post from informants that give rise to misperceptions in sharenting upload responses, for example in $\mathrm{AB}$. This informant uploaded the childbirth content after 9 months of pregnancy, but there were followers who thought the child from $A B$ died after 9 months. In addition to misperceptions in AB's response, the informant revealed that her followers prayed for positive things as responses. Informants get more responses when they upload about their children than upload about themselves.

\section{DISCUSSION}

Before this study limited the focus on Instagram as social media used as sharenting media by informants, this study acknowledged that there were other media that became a place of sharenting activity. Mothers also did sharenting on several alternative media, including WhatsApp Status, family groups, Facebook, and Path. Instagram is the main social media as a mother sharenting container, but other media are used as an alternative to limiting sharenting to the closest people. WhatsApp family group is an alternative for sharing photos and videos because it is limited to families. Another finding is that sharenting is not only done by mothers, but also done by close relatives. Uploading children's photos and videos are also done by fathers, uncles, and grandparents. This is related to situations in which informants are together with close relatives when caring for or playing with children, so 
that the family participates in documenting the moment of the child and uploads the photo or video. The next finding is about rules that are owned by each informant in doing sharenting. Rules that are applied are in the form of not sharing too many photos of children's faces on Instagram, not being too showy and excessive in distributing children's activities, not uploading children's photos with uniforms or school attributes, not showing off children who have the potential to make others sad; and only upload photos of children in proper pose: not naked, etc.

Mother constructs sharenting reality through three recurring phases: externalization, objectivation, and internalization. These three phases involve three reality perspectives, namely objective reality, symbolic reality, and subjective reality. The construction of sharenting reality done by mothers is not only done offline, but also done online through Instagram. The process of reality construction carried out online is explained using the Crystallization concept which describes the three phases of reality construction in the era of social media. The objectivation phase occurs in mothers when getting information on Instagram from public figures, professional psychologists, and fellow mothers who also do sharenting. The internalization phase occurs when the information obtained by the mother, both online and offline, then constructs the mother's view of positive and negative values in sharenting activities, which is then reflected in sharenting upload photos or videos when the mother adjusts to her environment. The externalization phase occurs when mothers can adjust to objectivation and internalization carried out subjectively based on sharenting goals and making rules on content that may or may not be shared. The three phases of sharenting reality construction are illustrated in the sharenting process that we identified into six stages: (1) documentation, (2) choosing, (3) editing, (4) giving captions, (5) uploading, and (6) getting responses. These stages are not entirely carried out by each informant. This is a form of adjustment made by informants through the three phases of reality construction so that informants can determine which ones are necessary and unnecessary when doing sharenting and see sharenting as a positive activity by avoiding negative things that can be caused by sharenting.

\section{CONCLUSION}

Mother constructs sharenting reality through three recurring phases: externalization, objectivation, and internalization. The construction of sharenting reality done by mothers is not only done offline, but also done online through Instagram social media. The process of reality construction carried out online is explained using the crystallization concept which describes the three phases of reality construction in the era of social media. The three phases of sharenting reality construction are illustrated in the sharenting process that we identified into six stages: (1) documentation, (2) selection, (3) editing, (4) giving captions, (5) uploading, and (6) getting responses. In addition to the sharenting process, we also found sharenting rules of content that is allowed and appropriate to be shared on social media, positive and negative values for sharenting activities, family members other than mothers who also do sharenting activities, and other media that are also used for sharenting.

\section{REFERENCES}

[1] Brosch, A. (2016). When the Child is Born into the Internet: Sharenting as a Growing Trend among Parents on Facebook. The New Educational Review2, $43 \quad$ (1), 225-235. https://doi.org/10.15804/tner.2016.43.1.19

[2] Kumar, P., \& Schoenebeck, S. (2015). The ModernDay Baby Book: Enacting Good Mothering and Stewarding Privacy on Facebook. In Proceedings of the 18th ACM Conference on Computer Supported Cooperative Work (pp. 1302-1312). https://doi.org/10.1145/2675133.2675149

[3] Children Hospital University of Michigan Health System. (2015). Parents on Social Media Likes and Dislikes of Sharenting. National Poll on Children's Health (Vol. 23).

[4] Blum-Ross, A., \& Livingstone, S. (2017). "Sharenting," parent blogging, and the boundaries of the digital self. Popular Communication, 15(2), 110125.

[5] Autenrieth, Ulla (2018). Family photography in a networked age. Anti-sharenting as a reaction to risk assessment and behaviour adoption. p. 219-231 in Giovanna Mascheroni, Cristina Ponte \& Ana Jorge (eds.) Digital Parenting. The Challenges for Families in the Digital Age. Göteborg: Nordicom.

[6] Choi, G. Y., \& Lewallen, J. (2017). "Say Instagram, Kids!': Examining Sharenting and Children's Digital Representations on Instagram. Howard Journal of Communications, 29(2), 144-164.

[7] Bannon, S., Ford, K., \& Meltzer, L. (2011). Understanding millennials in the workplace. The CPA Journal, 81(11), 61-65.

[8] Damkjaer, M. S. (2018). Sharenting= Good Parenting? Four Parental Approaches to Sharenting on Facebook. In Digital Parenting. The International 
Clearinghouse on Children, Youth and Media at NORDICOM.

[9] Nugraha. (2019, February 22). Alasan Melakukan Sharenting. (N. R. Putri, Interviewer)

[10] Gaëlle, O., \& Verswijvel, K. (2018). Sharenting: Parental adoration or public humiliation? A focus group study on adolescents' experiences with sharenting against the background of their own impression management. Children and Youth Services Review, 99, 319-327.

[11] Lichtenstein, F. (2017). Growing up on YouTube-How family vloggers are establishing their children's digital footprints for them.

[12] Jiow, H. J., Lim, S. S., \& Lin, J. (2016). Level up! Refreshing parental mediation theory for our digital media landscape. Communication Theory, 27(3), 309328.
[13] Collette, J. (2005). What kind of mother am I? Impression management and the social construction of motherhood. Symbolic Interaction, 28(3)

[14] Steinberg, S. B. (2017). Sharenting: children's privacy in the age of social media. Emory LJ, 66, 839.

[15] Berger, P. L., \& Luckmann, T. (1991). The social construction of reality: A treatise in the sociology of knowledge. Penguin UK.

[16] Wohn, D. Y., \& Bowe, B. J. (2014). Crystallization: How social media facilitates social construction of reality. In Proceedings of the companion publication of the 17th ACM conference on Computer supported cooperative work \& social computing (pp. 261-264). ACM.

[17] Patton, M. Q. (2002). Qualitative Research \& Evaluation Methods. Thousand Oaks, California: Sage Publications. 